\title{
Review of: "The effectiveness of diaphragmatic breathing relaxation training for improving sleep quality among nursing staff during the COVID-19 outbreak: a before and after study"
}

\section{Elsa Vitale}

Potential competing interests: The author(s) declared that no potential competing interests exist.

The manuscript is well written, well structured, clear: congratulations to the authors.

The only detail to improve: the ethical considerations: both in the methodology and at the end of the manuscript three sentences are presented about it. Personally, at the end of the manuscript I would put the relevant references of the Ethics Committee both for the approval of the study and for the approval of the protocol. For the rest, I do not think there are any further changes. 